\title{
Crack Analysis in Single Plate Stressing of Particle Compounds
}

\author{
Manoj Khanal, Wolfgang Schubert, and Jürgen Tomas \\ Mechanical Process Engineering, Process Engineering Department, \\ Otto-von-Guericke-University of Magdeburg, D-39106 Magdeburg, Germany \\ \{Manoj.Khanal, Wolfgang.Schubert, Jürgen.Tomas\} @vst.uni- \\ magdeburg. de
}

\begin{abstract}
Particle compound material is the composition of different particles with inhomogeneous and non-uniform properties. Particle compound material is the most complicated engineering material whose properties vary according to the applications, method of manufacturing and ratio of its ingredients. The quasi-homogeneous materials like building materials and constituents of tablets, pellets etc are some of the examples of particle compounds.

The 2 Dimensional Finite Element Analysis was done with single plate compressive stressing on the particle compound material to have the idea of the stress distributions during stressing. Then the method was followed by the Discrete Element Method (DEM) for further analysis.

The study of crack propagating mechanism in particle compound was represented by a model material which resembles the high strength materials, pressed agglomerates and more. The paper analyses the cracking of the particle compounds, here concrete ball, with respect to continuum and discrete approach.
\end{abstract}

\section{Introduction}

Particle compound material [1] is the composition of different particles with inhomogeneous and non-uniform properties. The research with the spherical shaped particle compound, here concrete sphere, was to observe the cracking mechanisms while subjected to single plate stressing along the diameter during diametrical compression test. The aggregate material as the value component is fixedly embedded in concrete so that cracking can only occur by forced crushing. During this process the bonds between aggregate and binding material, which is the second but valueless component, have to be burst. The compression stressing test, termed as indirect tensile test, can be considered as one of the method to evaluate the fracture strength of particle compounds.

There are number of references by Schubert [2], Kiss and Schönert [3], Arbiter et al. [4], Tomas et al. [5], Potapov and Campbell [6], Mishra et al. [7], Salman et al. [8], Moreno et al. [9] and Khanal et al. [10] which have shown the study of impact and compression stressing for the fracturing of materials and cracking of material during normal and oblique impact conditions. Khanal et al. [10] have studied the central and oblique impact of particle compound materials with finite and discrete element methods and compared with experiments of central impact condition. The discrete 
element simulations of diametrical compression tests were carried out [11,12,13]. Thornton et al. [11] have studied the diametrical compression simulation on dense and loosely packed agglomerates and showed that the loose agglomerates failed by disintegration and dense agglomerate by fractured. For the low strength materials like tablets, the simulations/measurements are generally performed with comparatively low velocity for slow deformation, quasi-static, where the test specimen fails by propagation of one or few cracks at a failure stress that is relatively insensitive to the loading rate [13] (see[11]). The experiment and analysis of fragmentation of brittle spheres under static and dynamic compressions were dealt by Chau et al. [14]. The finite element method has also been adopted to study the crushing of spherical materials $[15,16]$.

The cracking method in spherical model is different than the regular structures when subjected to impact or diametrical stressing loading. To study the cracking mechanism in the concrete ball, the two-dimensional Finite Element compressive analyses were carried out for single plate diametrical stressing condition to understand the stress pattern distributions before cracking. In reality, the discrete element approach is preferable for the analysis of the particle compound, particularly to understand the fragmentation behaviour during fracturing, which treats the particle compound as the constituents of different individual small balls as the particles. The compression stressing on concrete sphere is done with relatively higher speed with higher deformation rate as compared to the agglomerates.

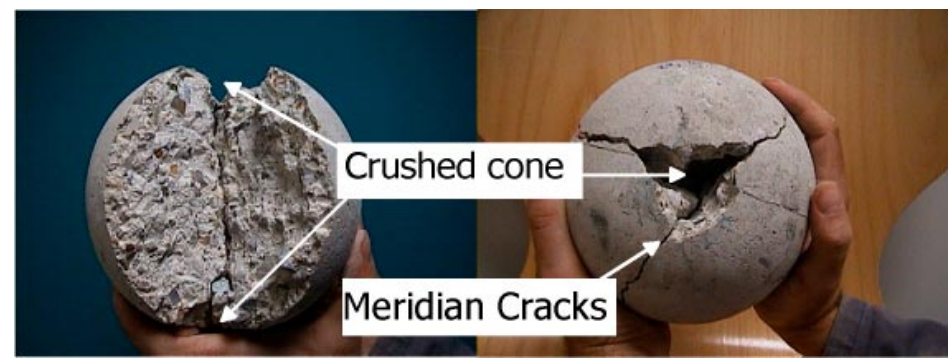

Fig. 1. Cracks observed during single plate stressing of concrete sphere.

\section{Results and Discussions}

\subsection{Experimental}

Spherical shaped concrete spheres of $150 \mathrm{~mm}$ in diameter of B35 $\left(35 \mathrm{~N} / \mathrm{mm}^{2}\right.$ compressive strength) strength category as common material of civil engineering were chosen for the single plate compression stressing. The compression stressing experiments were done with universal testing machine. The concrete sphere was clamped on the lower base plate and the upper plate was moving with the defined velocity to generate the stress inside the sphere. The concrete sphere was coloured with different colours before crushing. After crushing, the generated fragments were collected, assembled according to the colour and analyzed to observe the crack 
patterns. The fractured ball obtained from experiment is shown in Fig.1 with meridian cracks and crushed cone.

\subsection{Finite Element Simulation of Compression Test by Single Plate Stressing}

To study the dynamic stress patterns generated inside the concrete ball during single plate diametrical stressing, the finite element simulation was carried out with commercially available software called ANSYS [17]. Though the continuum analysis does not allow to visualize the high velocity multiple crack propagation and fragmentation behaviour during simulation, but it provides the idea of stress distributions inside the sphere during stressing. It is known that crack generation and propagation are functions of stress [1]. Hence, the finite element analysis can be considered as an essential investigation. The finite element model description with input parameters is dealt in another paper [1]. An ANSYS model was considered for a two-dimensional sphere of $150 \mathrm{~mm}$ in diameter, stressing at the velocity of $20 \mathrm{~m} / \mathrm{s}$. Actually, different velocities were tried and it was noted that the change in velocities causes the change in intensity of stresses. Hence, for the clarity here the simulation of only one velocity is presented.

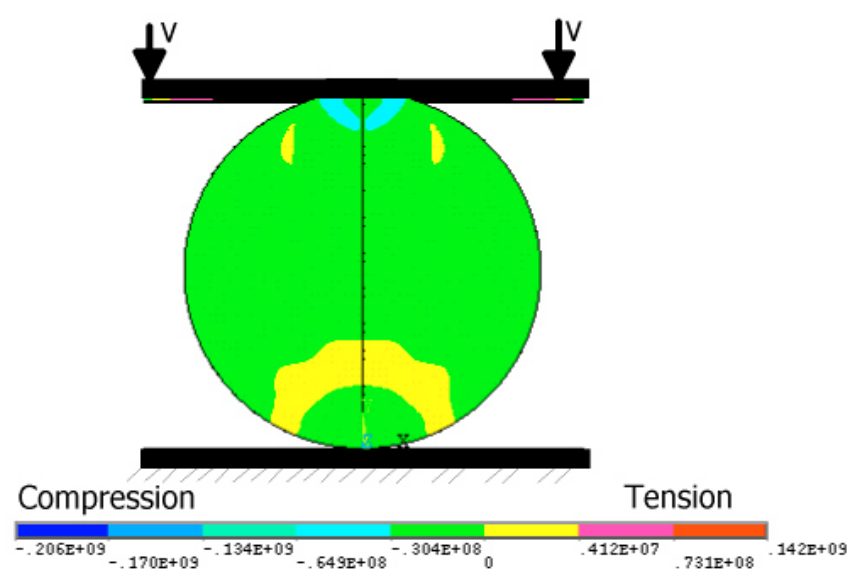

Fig. 2. Stress generated in stressing direction during compression test.

Fig 2. shows the stress generated during single plate diametrical stressing. The upper plate is stressing the ball and lower plate is constraint from any movement. When the plate stresses the ball, the stress wave is initiated and propagated from the contact zone and moves towards the other low stressed zone (opposite side). These different stressed zones show distinct region nearer to the contact zone and is similar to the cone in three dimension or wedge (triangle) in two dimension. At the contact area the compressive zones can be seen as a triangular shape. The tension waves are generated from the boundary of the contact zone and can be seen in figure as two dynamic tension spots nearer to the contact zone. The propagated tension waves can also be seen on the fixed plate side as an arch shape. The boundary of the conical or wedge shape has the maximum absolute change in values from compression to tension. This transition from the compressive to tensile region takes place in the 
periphery of the compressed zone. Hence, this region has a dominant effect on the crack generation, and so, the boundary of this region initiates the crack with a similar shape during stressing. In single plate stressing, though the stressing is done in one direction, the other direction has also the similar disturbed region like the shape of the wedge. This is because of the propagation and reflection of the waves in to and fro direction. This implies even in single plate compression, there should be the formation of the another wedge in opposite side (i.e. in the stationary plate side). The dimension of this second wedge depends upon the stressing condition and velocity. The generation of two wedges can be further verified by discrete element simulations. The propagating tension waves from stressing to non-stressing sides should also generate the cracks in the similar propagating manner from one side to another. These types of cracks are called meridian cracks in experiments and diametrical cracks in 2 dimensional simulations.

\subsection{Discrete Element Simulation of Compression Test by Single Plate Stressing}

The concrete sphere is considered as the mixtures of different sized particles having random properties with porosities. Though the material seems complicated, it can be easily modelled with the principle of discrete element method. The discrete element solution scheme assumes each of its constituents as the separate entity and applies the Newton's second law of motion and force displacement law at contacts. This individual particles allow to delete the contact when they experiences the higher force than their material strength. This process shows the fragmentation behaviour of the material, like in reality. The two dimensional concrete ball was modelled with randomly arranged 1000 particles as distinct elements with the PFC [18] which solves with distinct element method. The detailed modelling of concrete ball and input parameters are dealt in [1]. Left picture of Fig 3. shows the assembly of gravels (aggregates) and hardened cement paste to represent the concrete ball. Thus modelled concrete ball was diametrically compressed with single plate stressing. In single plate stressing, the upper plate was allowed to move against the ball and the lower plate was fixed.
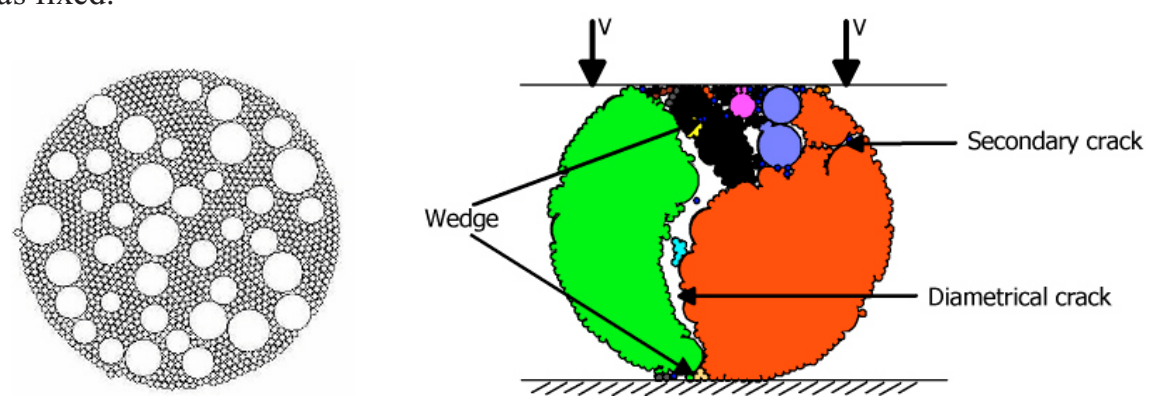

Fig. 3. Left - modelled concrete ball with DEM (bigger balls - aggregates, smaller balls hardened cement paste), Right - Fractured ball during compression test by single plate stressing.

Right picture of Fig. 3 shows the fractured ball during single plate compression at the plate velocity of $1 \mathrm{~m} / \mathrm{s}$. The different gray tone pieces show the fragments 
generated after crushing. Here, two disturbed regions are generated in two sides of the ball - one on the stressing side and another on the fixed plate side. In the stressing side, the crushed region has the similar wedge shape as predicted by continuum analysis. The crushed wedge in the stationary plate side is small as compared to the case of stressing side. The stress waves generated during stressing are somewhat damped on the boundary of the particles and on the discontinuous region of the material and only the less amount of the stress waves propagate and reach in the lower side of the ball. Hence, this causes the less disturbances as compared to the stressing side, as a result, the smaller wedge is produced in the opposite side of the stressing. The figure shows the different types of cracks obtained during simulation. The diametrical cracks are propagated from stressed side to the low stressed side. The secondary cracks are linking the diametrical cracks. The bigger balls on the crushed zone show the liberated aggregates during stressing.

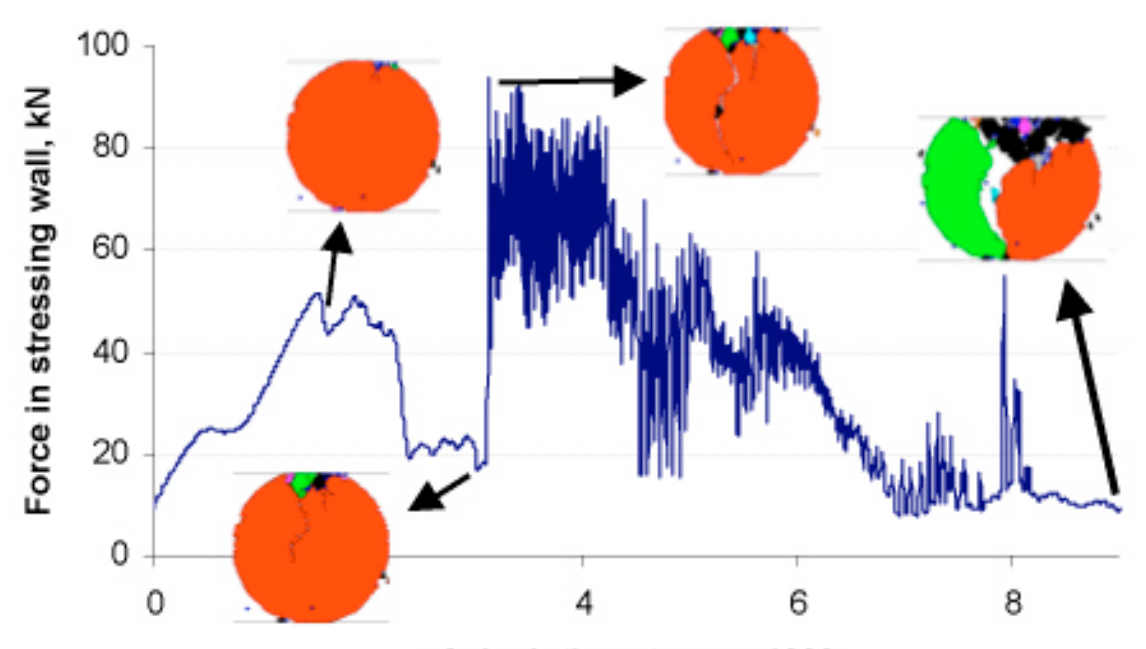

Calculation steps, x 1000

Fig. 4. Different stages of fractured ball during single plate compression stressing, $v=1 \mathrm{~m} / \mathrm{s}$.

The different stages during compression test by single plate stressing of two dimensional concrete sphere with calculation steps are shown in Fig. 4. The first peak was observed when there was a starting of the crack. The fall in force curve after the first crack shows that the energy was utilized to generate the crack. The generation of crack means the generation of new surfaces and consumes energy. It is clear from the figure that before the generation of cracks, the force curves are increasing and after propagation of the cracks these force curves are decreasing. This shows that energy is required to generate and propagate cracks. In other words, crack propagation is the generation of new surfaces. It is seen from the figure that even after failure of the ball the force curves attain the higher values. The reason for this is, when wall touches the aggregates, the wall experiences the higher amount of resistance from the aggregates (bigger balls). In other cases, the disturbances caused by the stressing of wall will be adjusted by the discontinuous nature of the material. Which means, the stress waves 
propagating inside the ball from the stressing condition will be damped by the boundaries of the particles and the pores present in-between the constituents of the concrete spheres.

\subsection{Comparison of Experiments and Simulations}

Fractured ball from experiment and simulations showed the crushed cone (wedge in 2D simulation) during stressing. In the experimentally fractured ball, the inelastic contact deformation produces the cone of fines [5]. The contact area was noted very smooth, all the micro irregularities were smoothened. There were no secondary cracks generated during experiments. This is because it was not allowed to crush further after the breaking of the ball. This provides the evidence that the secondary cracks are generated only after forming the meridian cracks. The experiments were conducted with the velocity of $1 \mathrm{e}-6 \mathrm{~m} / \mathrm{s}$, which is obviously very less stressing velocity to generate the secondary cracks. This experiment was done with Universal Testing Machine (UTM), where it was impossible to provide higher velocities. Even if the stressing plate was allowed to stress further, the plate motion would not be sufficient to hold the ball from falling down from the lower plate to crush it because immediately after fracturing the ball, the ball crushed into pieces and fell out from the experimental rig due to gravity. But in discrete element simulation it was possible to farther the stressing wall against the ball. Hence, we can see the secondary cracks in the fractured ball. This also adds to the proof that the secondary cracks are generated only if there is sufficient energy remaining after forming the meridian cracks. Here, it has to be noted that the experiments are 3 dimensional whereas the simulations are 2 dimensional, that is why it is not possible to relate point to point between them.

\subsection{New Surface Generation}

It is known that during stressing of the sphere, the fracturing process produces the fragments and the quantity of fragments depends upon the stressing condition. Here, the stressing condition imply the stressing velocity and the material strength of the model. In discrete element method all the constituents are glued together with the bonds existing between the particles. Therefore, when these bonds break the particles are separated out. In other words, the breaking of bonds mean the generation of the new surfaces. Fig. 5 shows how the bonds are breaking with the increasing velocity. From figure, it can be observed that with the increasing velocity the bond breakage between the particles are also increasing. Actually, the broken bonds are nothing it is similar to producing the cracks. When the material is not able to sustain the strength generated by stressing, the material deletes the contact with each other and propagates the crack. For the less stressing energy less bonds between the particles are broken and with higher velocities more bonds are broken and after certain limit of the stressing velocity no further more bonds are broken. Hence, higher than this limiting velocity there is no production of broken bonds even with the increasing stressing velocities. Here, in this case, the effective number of bonds are breaking at the range of $5-10 \mathrm{~m} / \mathrm{s}$. After $10 \mathrm{~m} / \mathrm{s}$ the process becomes inefficient because there will not be any significant number of bonds breaking even with increasing input energy. The application of the input energy after this limiting velocity is a wastage of the energy. 
This can be validated by the new surface generation curve (dotted curve) as shown in figure, which shows that the new surface generating process is efficient till $10 \mathrm{~m} / \mathrm{s}$.

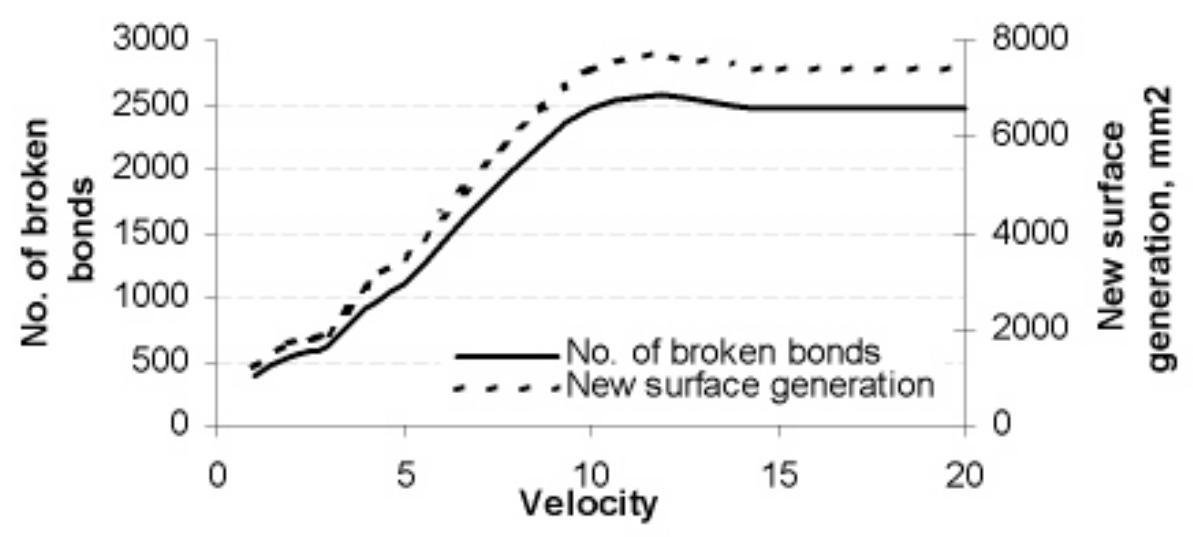

Fig. 5. Broken bonds and new surface generation versus velocity.

\section{Conclusions}

The stress pattern distributions obtained from the finite element simulation during stressing was the criterion to predict the crack initiation and propagation in the spherical particle compound materials. The formation of the wedge predicted by finite element method was further verified from the discrete element method. Discrete element analysis showed the crack initiation and propagation in the similar way as predicted by finite element simulation along with generation of different cracks and thus forming different fragments. The secondary cracks were clearly seen and analyzed with discrete element analysis.

The discrete element analysis was done with 2 dimensional software. It has presented the basics of crushing and fragmentation phenomena with less time. But there were some limitations in $2 \mathrm{D}$ simulation, as the crushed cone could not be seen as cone instead it can be seen as wedge (triangle). Hence, during simulation it was realized that 3 dimensional software could provide realistic inside to the crushing system to analyse the cracking mechanism.

Acknowledgement. The authors would like to acknowledge German Research Foundation (DFG) for the financial support. 


\section{References}

1. M. Khanal, W. Schubert, J. Tomas, Ball Impact and Crack Propagation - Simulations of Particle Compound Material, Granul. Matter, 5 (2004) 177-184.

2. H. Schubert, Zur Energieausnutzung bei Zerkleinerungsprozessen, Aufbereit. - Tech. 34 (10) (1993) 495-505

3. L. Kiss, K. Schönrt, Aufschlusszerkleinerung eines zweikomponentigen Modellstoffes unter einzelkornbeanspruchjung durch druck-rund prallbeanspruchjung [Liberation of two component materail by single particle compression and Impact crushing]. Aufbereit.-Tech.30(5)(1980)223-230

4. N. Arbiter, C. C. Harris, G. A. Stamboltzis, Single fracture of brittle spheres, Soc. Min. Eng. AIME, Trans. 244 (1969) 118-133

5. J. Tomas, M. Schreier, T. Gröger, S. Ehlers, Impact crushing of concrete for liberation and recycling, Powder Technol 105 (1999) 39-51

6. Alexander V Potapov, Charles S. Campbell, Computer simulation of impact-induced particle breakage, Power Techno, 82 (1994) 207-216

7. B. K. Mishra, C. Thornton, Impact breakage of a particle agglomerates, Int. J. Miner. Process. 61 (2001) 225-239

8. A. D. Salman, D. A. Gorham, A. Verba, A study of solid particle failure under normal and oblique impact, Wear 186-187 (1995) 92-98

9. R. Moreno, M. Gadhiri, S. J. Antony, Effect of impact angle on the breakage of agglomerates: a numerical study using DEM, Powder Techno, 130 (2003) 132-137

10. M. Khanal, W. Schubert, J. Tomas, Crack initiation and propagation - Central and oblique impacts, Comp. Mater. Sci. (submitted)

11. Thornton, M. T. Ciomocos, M. J. Adams, Numerical simulations of diametrical compression tests on agglomerates, (obtained per mail)

12. A. Lavrov, A. Vervoort, M. Wevers, J A L Napier, Experimental and numerical study of the Kaiser effect in cyclic Brazilian tests with disk rotation, Int. J. Rock Mech. Min., 39 (2002) 287-302

13. Malcom Mellor, Ivor Hawkes, Measurement of tensile strength by diametrical compression of discs and annuli, Eng. Geol., Vol, 5, Issue 3 (1971) 173-225

14. K. T. Chau, X. X. Wei, R. H. C. Wong, T. X. Yu, Fragmentation of brittle spheres under static and dynamic compressions: experiments and analyses, Mech. Mater., 32(2000) 543-554

15. S. Lee, G. Ravichandran, Crack initiation in brittle solids under multiaxial compression, Eng. Eng. Fract. Mech., 10 (2003) 1645-1658

16. Oliver Tsoungui, Denis Vallet, Jean Claude Charmet, Numerical model of crushing of grains inside two dimensional granular materials, Powder technology, 105 (1999) 1901998

17. FEM Manual ANSYS 6.1, ANSYS Inc., Southpointe, 275 Technology Drive, Canonsburg, PA 15317.

18. Particle Flow Code in 2 Dimensions Manual, Vers. 3.0, Itasca Consulting Group Inc., Minneapolis, Minnesota, US (2002). 